\title{
IFSO Endoscopy Committee Position Statement on the Practice of Bariatric Endoscopy During the COVID-19 Pandemic
}

\author{
Christine Stier ${ }^{1} \cdot$ Gontrand Lopez-Nava $^{2} \cdot$ Manoel Galvao Neto ${ }^{3} \cdot$ Christopher C. Thompson $^{4} \cdot$ Josemberg Campos $^{5}$. \\ Mousa Khoursheed ${ }^{6} \cdot$ Muffazal Lakdawala $^{7} \cdot$ Almino Ramos $^{8} \cdot$ Barham K. Abu Dayyeh $^{9}$ (D
}

Published online: 4 July 2020

(C) Springer Science+Business Media, LLC, part of Springer Nature 2020

\begin{abstract}
COVID-19 (Coronavirus disease 2019) caused by SARS-CoV-2 has become a global pandemic. Obesity is a risk factor for severe disease, and the practice of endoscopy poses special challenges and risks of SARS-CoV-2 transmission to patients and providers given the evolving role of the gastrointestinal tract in viral transmission and aerosol generation during endoscopic procedures. It is therefore necessary to distinguish between urgent interventions that cannot be postponed despite the risks during the pandemic and, in contrast, purely elective interventions that could be deferred in order to minimize transmission risks during a time of infection surge and limited access. Semi-urgent bariatric procedures have an intermediate position. Since the chronological course of the pandemic is still unpredictable, these interventions were defined according to whether or not they should be performed within a nominal 8-week period. In this position statement, the IFSO Endoscopy Committee offers guidance on navigating bariatric endoscopic procedures in patients with obesity during the COVID-19 pandemic, in the hope of mitigating the risk of SARS-CoV-2 transmission to vulnerable patients and healthcare workers. These recommendations may evolve as the pandemic progresses.
\end{abstract}

Keywords Endoscopy $\cdot$ Bariatric $\cdot$ COVID-19 $\cdot$ Obesity $\cdot$ Obesity surgery $\cdot$ Bariatric endoscopy

Barham K. Abu Dayyeh

Abudayyeh.barham@mayo.edu

1 Sana Hospitals Germany, Obesity Center North Rhine-Westphalia, Department of General-, Visceral-, and Transplant Surgery, RWTH Aachen University, Aachen, Germany

2 Bariatric Endoscopy Unit at HM Sanchinarro University Hospital in Madrid, Madrid, Spain

3 FMABC, Santo Andre, Sao Paulo, Brazil

4 Division of Gastroenterology, Brigham and Women's Hospital, Boston, MA, USA

5 Federal University of Pernambuco, Recife, Brazil

6 Department of Surgery, Kuwait University, Kuwait City, Kuwait

7 Institute of Minimal Access Surgical Sciences and Research Centre, Saifee Hospital, Mumbai, India

8 Gastro-Obeso-Center Institute, Sao Paulo, Brazil

9 Division of Gastroenterology and Hepatology, Mayo Clinic, Rochester, MN, USA

\section{Position Statement}

COVID-19 (Coronavirus disease 2019) caused by SARSCoV-2 has become a global pandemic. The World Health Organization (WHO) declared COVID-19 as a pandemic on 11 March 2020. Up until 23 April, 2,649,680 cases have been reported in 185 countries [https://coronavirus.jhu.edu/map. $\mathrm{html}$. Studies showed that typical clinical symptoms included cough, sore throat, fever, fatigue, and shortness of breath [1]. So far, pulmonary manifestations, including interstitial pneumonia, which in its severe course may lead to lung failure and acute respiratory distress syndrome (ARDS/SARS), are the predominant source of morbidity and mortality. However, extrapulmonary manifestations of COVID-19 have been reported with an evolving understanding of their contribution to disease transmission and morbidity. In a Chinese study, up to $27.8 \%$ of patients have myocardial injuries that can lead to cardiac dysfunction and arrhythmias [2]. In addition, Covid-19 has been shown to cause multiple gastrointestinal symptoms with $5 \%$ of patients having nausea or vomiting and $3.8-10.1 \%$ having diarrhea [3]. A 
recent Chinese study showed that more than half of patients (53.42\%; 39/73, tested within 14 days) tested positive for SARS-CoV-2 RNA in stool [4]. Furthermore, SARS-CoV-2 binds angiotensin-converting enzyme 2 (ACE2) protein of the host cell membrane to fuse into the cell for nucleic acid replication; thus, organs expressing the ACE2 protein have been implicated in viral transmission. Immunofluorescence data demonstrated that ACE2 is abundantly expressed in salivary, gastric, duodenal, and rectal epithelia, in addition to hepatic cholangiocytes and pancreas endocrine and exocrine cells, implicating the gastrointestinal tract in COVID-19-related morbidities and SARS-CoV-2 transmission [4-6].

The practice of endoscopy poses special challenges and risks of SARS-CoV-2 transmission to patients and providers, given the evolving role of the gastrointestinal tract in viral transmission and aerosol generation during endoscopic procedures. While the transmission of SARS-CoV-2 through droplet infection by contact with infected persons is clear, the aerosol transmission of SARS-CoV-2 poses additional risks. Droplets are particles above $20 \mu \mathrm{m}$ in size. They are usually produced with coughs, sneezes, and shouting. Aerosols comprise fine particles under $10 \mu \mathrm{m}$. It is not yet clear how far the respective particles are transported in the environment, but it is assumed that aerosols can be moved more easily and further in the air [7]. One study reported that the virus can remain viable and infectious in aerosols for hours and on surfaces for up to 3 days [8]. Furthermore, particles smaller than $5 \mu \mathrm{m}$ can migrate directly into the alveoli, whereas the path of particles larger than $10 \mu \mathrm{m}$ ends naturally below the glottis in the bronchial tree. This worldwide pandemic has led to a global lockdown in most countries with suspension of elective surgeries and endoscopic procedures during time of infections surge. Therefore, in addition to the protection measures for personnel, there is a need for a clear definition of which bariatric and metabolic endoscopic interventions are defined as time sensitive and should be carried out during a time of infections surge and which interventions can be postponed to minimize the risk of transmission to medical personnel. This is of critical importance, as the Centers for Disease Control and Prevention (CDC) have classified severe obesity (body mass index (BMI) of 40 or higher) and diabetes as risk factors for severe COVID-19 illness [https://www.cdc.gov/coronavirus/2019-ncov/need-extraprecautions/groups-at-higherrisk.html]. Furthermore, a large case series of sequentially hospitalized patients with confirmed COVID-19 in the USA showed that pre-existing hypertension and/or diabetes were highly prevalent in this cohort and that ventilated patients had high mortality rates [9].

Recommendations and guidelines are therefore needed to be well prepared to face this pandemic as bariatric endoscopists, in order to mitigate the risk of SARS-CoV2 transmission to our vulnerable patients and healthcare workers. To formulate our general recommendations, we relied on the recommendations of the Asian Pacific Society for Digestive Endoscopy (APSDE-COVID statements) [10] and the American Gastroenterological Association (AGA) institute rapid recommendations for gastrointestinal procedures during the COVID-19 pandemic [11]. Specific recommendations for bariatric endoscopy were empirically formulated based on expert opinion and extrapolated from the aforementioned guidelines.

\section{How Should the Bariatric Endoscopist Triage Procedures (Table 1)}

Both the APSDE and AGA guidelines call for deferment of elective endoscopies. Bariatric endoscopists are confronted with a wide spectrum of clinical situations where endoscopy could be deemed urgently required to manage a complication of bariatric surgery or remove an implanted bariatric device for treatment or prevention of a complication. Thus, we recommend that all bariatric endoscopy procedural requests be reviewed by an expert bariatric endoscopist and categorized as time sensitive, requiring endoscopy within 8 weeks or not during a time of infections surge. To make an appropriate determination about time sensitivity, consideration should be given to patients' symptoms, which should be ascertained via a telehealth visit when possible. Patients' engagement in decision-making and documentation of informed consent are critical components of the process.

With increased COVID-19 testing capabilities using both molecular (real-time PCR for active infection) and serologic (ELISA based for previous exposure and immunity), gradual access to semi-urgent and elective endoscopic procedures will increase (Fig. 1) [12]. Molecular testing has targeted a combination of the following SARS-CoV-2 genes: nucleocapsid $(\mathrm{N})$, open reading frame $1 \mathrm{ab}(\mathrm{Orf})$, envelope (E), and RNAdependent RNA polymerase (RdRp). Serologic testing detects IgG-class antibodies to SARS-CoV-2 that could be associated with neutralizing antibody activity and potential protective immunity but is not a replacement for molecular testing as a marker of acute infection. It is equally important to understand testing limitations. Peak viral shedding occurs $24 \mathrm{~h}$ prior to symptom onset [13], and molecular testing sensitivity using nasopharyngeal swabs drops 3-5 days post onset of symptoms or as more severe disease migrates into the lower respiratory tract; thus, bronchoalveolar lavage or sputum collection becomes more sensitive [14] (Table 2). For the serologic test, sensitivity has not yet been tested in patients with mild or asymptomatic infection; however, in hospitalized patients, sensitivity appears low $\leq 7$ days after symptom onset and high $\geq 14$ days. It is not known, however, if SARS-CoV-2 antibodies confer immunity or at what titer. Thus, it is important to clearly understand testing limitations influenced by the timing of collection, sample type, sample quality, and test 
Table 1 How should the bariatric endoscopist triage procedures?

\begin{tabular}{|c|l|l|}
\hline \multicolumn{1}{|c|}{$\begin{array}{c}\text { Elective Bariatric } \\
\text { Endoscopy }\end{array}$} & $\begin{array}{c}\text { Semi-Urgent Bariatric } \\
\text { Endoscopy }\end{array}$ & \multicolumn{1}{|c|}{$\begin{array}{c}\text { Urgent Bariatric } \\
\text { Endoscopy }\end{array}$} \\
\hline $\begin{array}{l}\text { No short-term impact on } \\
\text { patient-important } \\
\text { outcomes }\end{array}$ & $\begin{array}{l}\text { Delay can rapidly worsen } \\
\text { the patient's condition and } \\
\text { risks of delaying the } \\
\text { procedure are probably } \\
\text { higher the risks of SARS- } \\
\text { CoV-2 transmission in } \\
\text { appropriate setting an organ } \\
\text { the patient's life or results } \\
\text { in permanent dysfunction }\end{array}$ \\
\hline Delay $>\mathbf{8}$ weeks & $\begin{array}{l}\text { Perform within } \mathbf{4 8 h r s ~ t o ~} \leq \\
\mathbf{8} \text { weeks }\end{array}$ & Proceed without delay \\
\hline
\end{tabular}

performance, prior to developing a pre-procedural testing strategy. The likely optimal testing strategy will be a combination of molecular and serologic testing. The ramp-up period will probably vary by geographic location based on disease prevalence, testing capabilities, and availability of adequate personal protective equipment (PPE). Notably, a new SARSCoV-2 neutralizing antibody test, in addition to vaccine development efforts, might change the triage and access strategy to elective bariatric endoscopic procedures in the future.
Elective bariatric endoscopy procedures that should be delayed $>8$ weeks (GREEN) are as follows:

a. Primary bariatric intervention (implantable gastric and small intestinal devices, gastric remodeling techniques, aspiration therapy, duodenal resurfacing procedures).

b. Revisional bariatric intervention (transoral outlet reduction (TORe), restorative obesity surgery endoscopic (ROSE), endoscopic sleeve gastroplasty revision of laparoscopic sleeve gastrectomy (R-ESG)).

\section{Tests for SARS-CoV-2/COVID-19 and Potential Uses}

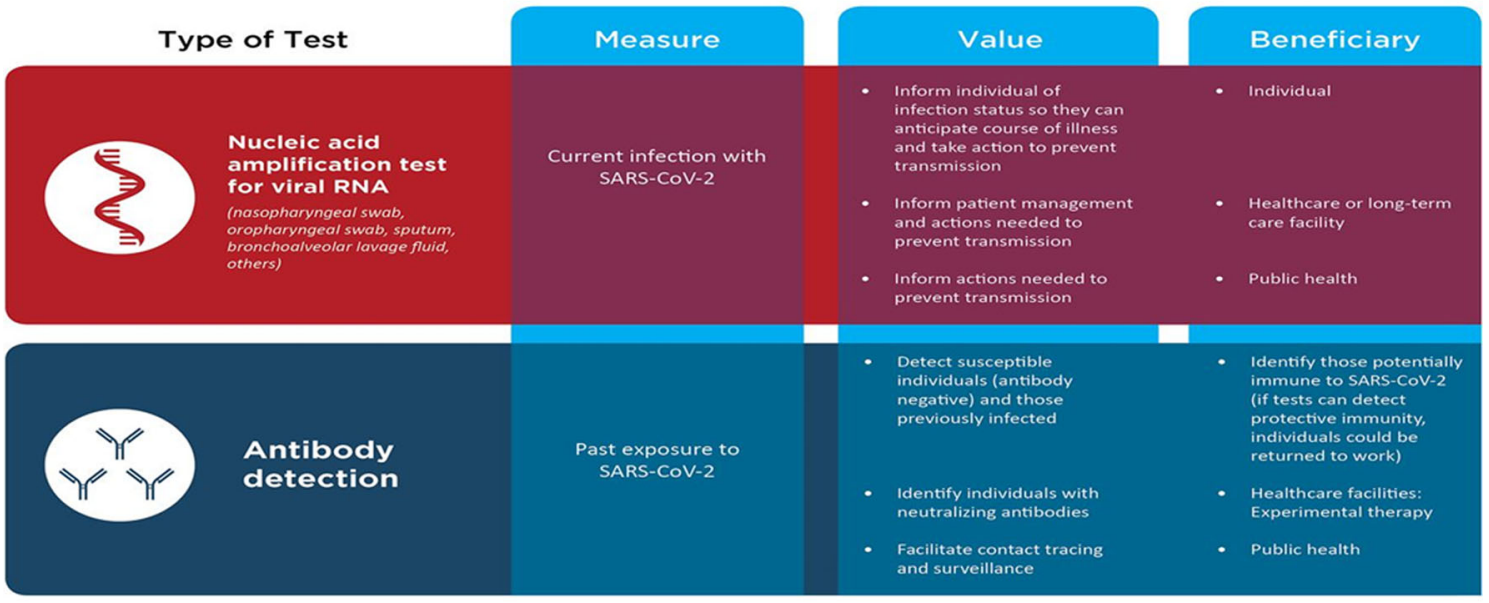

Robin Patel et al. mBio 2020; doi:10.1128/mBio.00722-20

Fig. 1 Tests for SARS-CoV-2 and potential uses. Adopted from Robin Patel et al. (mBio 2020) 
Table 2 Yield of different SAR-CoV-2 testing methods

Corona testing positivity rates- Journal of American Medical Association (AMA)

\begin{tabular}{lll}
\hline Si No & Type of specimen & Positive \% \\
\hline 1 & Bronchoalveolar lavage fluid & $93 \%$ \\
2 & Fibrobronchoscope brush biopsy & 46 \\
3 & Sputum & $72 \%$ \\
4 & Nasal swabs & $63 \%$ \\
5 & Pharyngeal swabs & $32 \%$ \\
6 & Feces & $29 \%$ \\
7 & Blood & $1 \%$ \\
8 & Urine & $0 \%$ \\
\hline
\end{tabular}

Adopted from Wang Xu et al. (2020)

c. Elective removal or adjustment of an implanted gastric or small intestinal device within the approved dwelling time of the device.

d. Elective removal of Orbera, Orbera 360 , or Spatz 3 intragastric balloon in asymptomatic patients in the period between 6 to 12 months after implantation. In the case of the Orbera IGB, delaying removal of the 6 months IGB to up to 9 months is acceptable during the pandemic period, if endoscopy access and resources are limited, based on the published literature describing safety, and requires careful patient counseling with periodic telemedical contact with the patient during the extended IGB dwelling time [15].

e. Elective upward adjustment of the Spatz3 intragastric balloon for enhancing weight loss.

f. Preoperative endoscopic examinations prior to bariatric surgery.

g. Follow-up endoscopic examinations for non-malignant conditions in asymptomatic patients such as nondysplastic Barrett's esophagus, esophagitis, gastritis, bile reflux, marginal ulceration, fistula, and gastrointestinal strictures.

h. Post-bariatric surgery endoscopic work-up of common complaints such as abdominal pain, gastroesophageal reflux, nausea, vomiting without clinical red flags, or documentation of pathology on radiographic imaging.

i. Elective removal or change of double pig-tail plastic stents that were previously placed for management of a chronic post-bariatric surgery leak and are in a suitable position without associated symptoms.

Semi-urgent bariatric endoscopy procedures that can be considered, provided appropriate informed consent is obtained, risk for SARS-CoV-2 transmission is minimized, and adequate availability of PPE or rapid reliable SARS-Cov-2 testing is ensured. These can proceed in $48 \mathrm{~h}$ to $\leq 8$ weeks (ORANGE).

a. Scheduled removal of any implantable gastric or small intestinal bariatric device at the manufacturer's recommended removal interval other than the Orbera IGB (see comments in the previous section).

b. Removal of any implantable gastric or small intestinal bariatric device for refractory symptoms.

c. Removal of an implantable gastric or small intestinal device during pregnancy. This should be a multidisciplinary team decision, coordinated and approved by obstetrics and or maternal fetal medicine.

d. Downward volume adjustment of the Spatz3 IGB to manage medically refractory symptoms.

e. TORe or ROSE procedure for treatment of early or late severe dumping syndrome refractory to medical management.

f. Endoscopic surveillance and management of preneoplastic or neoplastic conditions with significant risk of progression such as Barrett's esophagus with highgrade dysplasia or intramucosal cancer.

g. Removal or exchange of sponges for vacuum therapy of endoluminal bariatric leaks.

h. Removal or exchange of indwelling self-expanding or lumen-opposing metal stents to manage complications post-bariatric endoscopy or surgery, such as strictures and leaks.

i. Endoscopic management of symptomatic patients with documented anatomical post-bariatric surgery pathology by non-invasive imaging that can be addressed endoscopically, such as strictures.

j. Replacement of non-functioning or leaking A-tube for aspiration therapy.

Urgent bariatric endoscopy procedures that should be considered within $48 \mathrm{~h}$, to treat a condition that threatens the patient's life or results in permanent dysfunction of an organ. Upper or lower endoscopies in this setting should be considered high risk for SARS-Cov-2 transmission and require appropriate precautions and PPE or performance of rapid reliable SARS-Cov-2 testing (RED).

a. Removal of any intragastric device for symptoms of refractory gastric outlet obstruction symptoms, especially with presence of gastric dilation on non-invasive imaging.

b. Removal of migrated prosthesis or migrated/deflated gastric or small intestinal bariatric device within the reach of upper endoscopy. Of note, deflated IGB or double-pigtail plastic stents that have migrated to the small intestines (not within the reach of upper endoscopy) without 
symptoms of small bowel obstruction can be observed with serial abdominal imaging for self-excretion with defecation. Migration of any prosthesis or device into the small intestines with symptoms of small bowel obstruction will require emergency surgical consultation.

c. Endoscopic management of gastrointestinal bleeding, perforation, acute leaks, and severe stenosis.

d. Endoscopic management of a buried A-tube bumper during aspiration therapy.

\section{What Kinds of Precautions and PPE Are Needed for Urgent and Semi-urgent Bariatric Endoscopy Procedures}

All endoscopic procedures should be considered aerosolgenerating and high risk given the potential for coughing and retching during upper endoscopy and the passage of flatus during colonoscopy. In addition, all endoscopic procedures are at risk of generating aerosol and micro-droplets by the very

SEQUENCE FOR PUTTING ON PERSONAL PROTECTIVE EQUIPMENT (PPE)

The type of PPE used will vary based on the level of precautions required, such as standard and contact, droplet or airborne infection isolation precautions. The procedure for putting on and removing PPE should be tailored to the specific type of PPE.

\section{GOWN}

- Fully cover torso from neck to knees, arms to end of wrists, and wrap around the back

- Fasten in back of neck and waist
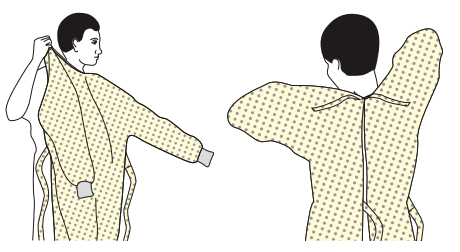

\section{MASK OR RESPIRATOR}

- Secure ties or elastic bands at middle of head and neck

- Fit flexible band to nose bridge

- Fit snug to face and below chin

- Fit-check respirator

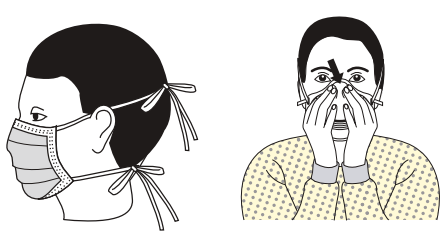

\section{GOGGLES OR FACE SHIELD}

- Place over face and eyes and adjust to fit

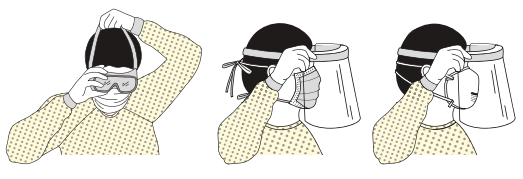

\section{GLOVES}

- Extend to cover wrist of isolation gown

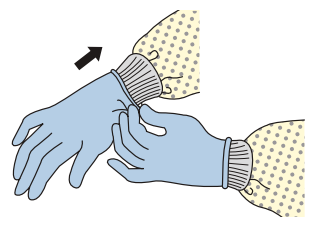

USE SAFE WORK PRACTICES TO PROTECT YOURSELF AND LIMIT THE SPREAD OF CONTAMINATION

- Keep hands away from face

- Limit surfaces touched

- Change gloves when torn or heavily contaminated

- Perform hand hygiene

Fig. 2 The correct sequence for wearing and removing PPE adopted from the CDC 


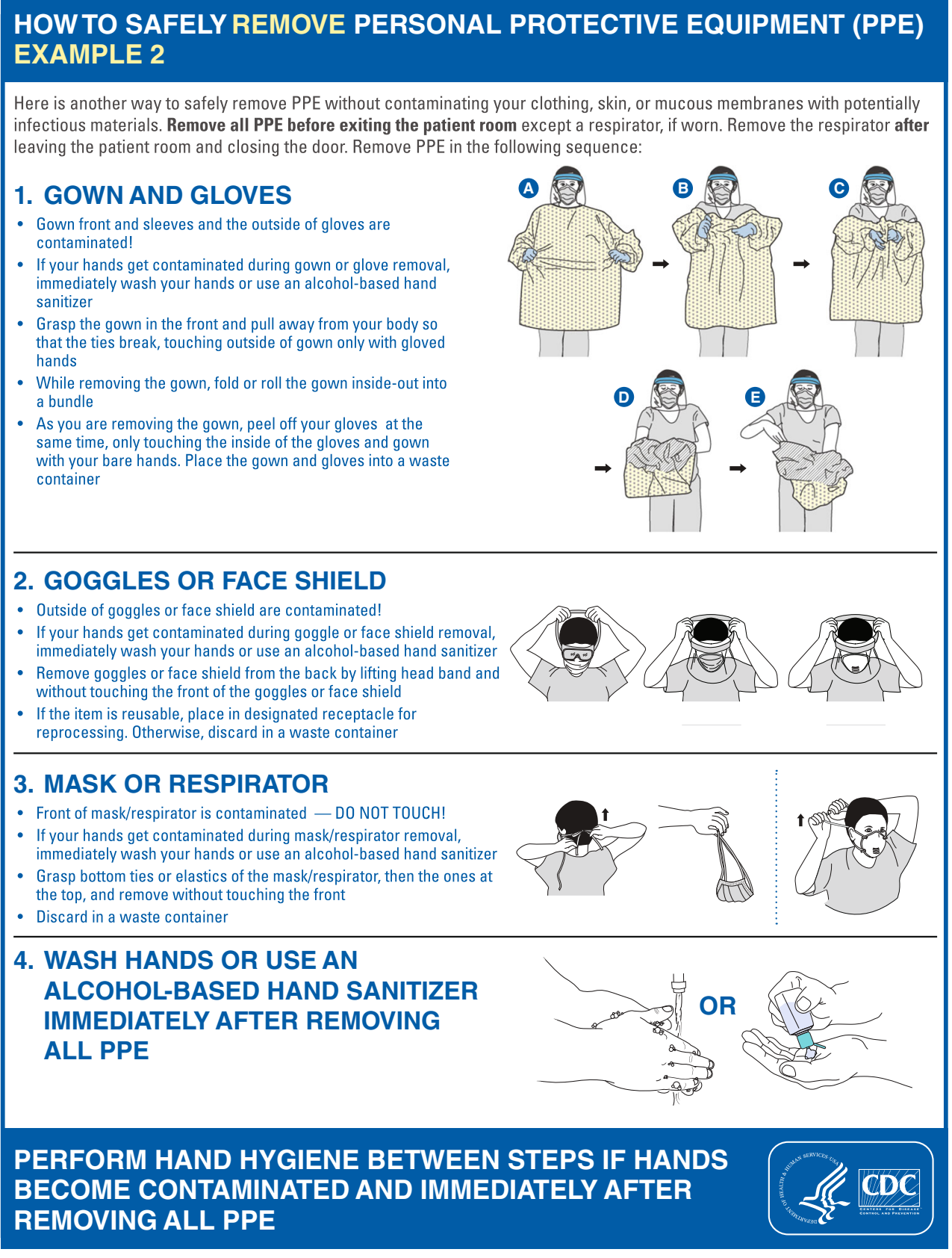

Fig. 2 (continued)

design, function, and leakage of the instruments, valves, ports, and air pressures during inflation and suction. The following measures are adopted from the US joint GI society statement on use of personal protective equipment in GI endoscopy during the COVID-19 pandemic [https://gi.org/2020/04/01/ joint-gi-society-message-on-ppe-during-covid-19].

a. General measures of physical distancing and adequate hand hygiene are of critical importance and need to be practiced diligently, independent of other protective measures.

b. All members of the endoscopy team should wear a full set of PPE, predicated on resource availabilities.

c. The correct sequence of wearing and removing PPE is critical and needs to be understood and practiced and instructions posted (Fig. 2) [16].

d. All members of the endoscopy team should wear N95 respirators (or devices with equivalent or higher 
filtration rates) for all GI procedures performed on patients with known SARS-CoV-2 infection and those with high risk of exposure. Given the high rate of infection transmission from pre-symptomatic individuals, all patients undergoing GI endoscopy in an area of community spread need to be considered "high risk" (Table 3).

e. All healthcare workers should have their N95 respirators fitted by an occupational health specialist prior to the first usage.

f. Staffing of endoscopy rooms should be reduced to the minimum number of individuals necessary to conserve PPE and other resources.

g. In some cases, shortages may require extended and limited reuse of N95 respirators. Guidance is available on how to wear, remove, and store respirators to minimize contamination [https://www.cdc.gov/niosh/topics/hcwcontrols/ recommendedguidanceextuse.html]. Decontamination of N95 respirators with hydrogen peroxide vapor has been approved by the US FDA as a means of reuse in times of limited supply [https://www.safety.duke.edu/sites/default/ files/N-95_VHP-Decon-Re-Use.pdf].

h. Use of Procedural Oxygen Masks (airway masks with apertures for endoscopes) should be considered for all upper endoscopy procedures to decrease aerosolization during these procedures. Intubation should also be considered in these cases to limit ongoing aerosolization. Topical spray anesthetics to numb the throat should be replaced in favor of a lidocaine swallow. Procedures should be performed in a negative pressure room when possible, especially for COVID-19 positive patients, or enough time between procedures should be allocated for complete air circulation and exchange [https://www. safety.duke.edu/sites/default/files/N-95 VHP-DeconRe-Use.pdf].

i. For endoscopes used on patients, regardless of COVID19 status, we recommend continuing standard cleaning endoscopic disinfection and reprocessing protocols.

j. It is important to recognize the limitations of available COVID-19 testing in your facility. According to a recent publication, nasal swabs, pharyngeal swabs, fecal testing, and bronchoalveolar lavage detected 63\%, 32\%, 29\%, and $93 \%$ of cases, respectively. Therefore, it is impossible to rely completely on any of these tests [14] (Table 2).

k. Adopting an incident response mentality is critical to endoscopy leadership during a time when physicians and staff are asked to embrace significantly altered workflows. Standard operating procedures must be established. There should be regular meetings of endoscopy leadership to review relevant information, with frequent scheduled updates provided to faculty and staff. Lastly, having an upstream communication channel to hospital leadership is critical, especially regarding information relevant to the safety of patients and staff [17].

Table 3 PPE for bariatric endoscopy during Covid-19 pandemic

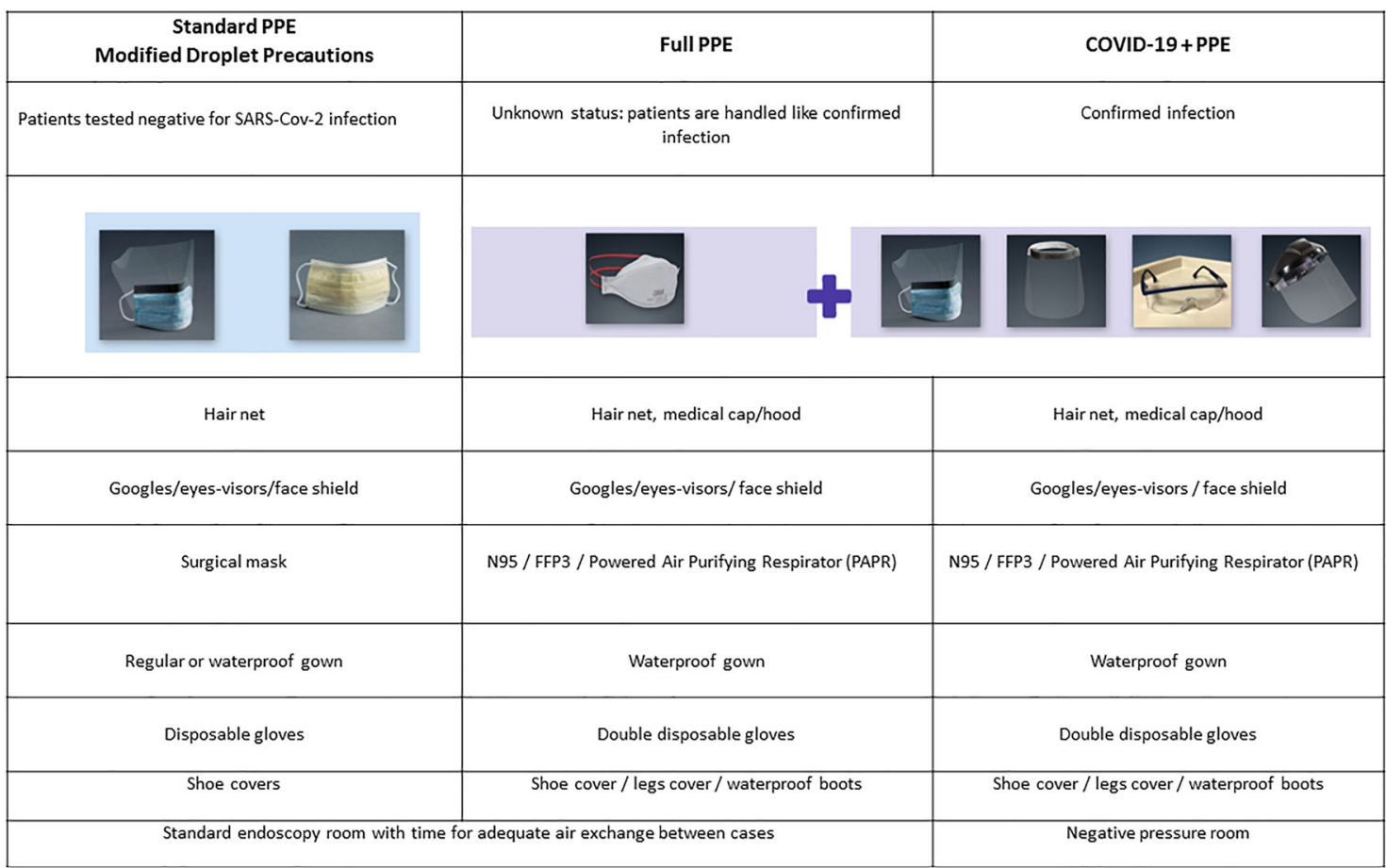




\section{Compliance with Ethical Standards}

Conflict of Interest Dr. Abu Dayyeh reports personal fees from Metamodix, BFKW, DyaMx, Boston Scientific, and USGI Medical; grants from Apollo Endosurgery, USGI, Spatz Medical, Boston Scientific, GI Dynamics, Cairn Diagnostics, Aspire Bariatrics, and Medtronic; and other from Johnson and Johnson and Endogastric Solutions. Dr. Lopez-Nava reports personal fees from Apollo Endosurgery, USGI, and Nitinotes. Dr. Galvao Neto reports personal fees from Apollo Endosurgery, USGI, and Colubris MX; personal fees from GI Dynamics and Keyron; and personal fees from Medtronics and Olympus, outside the submitted work. Dr. Thompson reports grants and personal fees from Apollo Endosurgery, grants from Aspire Bariatrics, others from BlueFlame Healthcare Venture Fund, grants and personal fees from Boston Scientific, personal fees from Covidien/Medtronic, personal fees and other from Fractyl, grants and personal fees from GI Dynamics and other from GI Windows, grants and personal fees from Olympus/Spiration, grants from Spatz, and grants, personal fees, and other from USGI Medical, outside the submitted work. Dr. Stier, Dr. Ramos, Dr. Campos, Dr. Khoursheed, Dr. Lakdawala, and Dr. Ramos had nothing to disclose.

Ethical Approval This article does not contain any studies with human participants or animals performed by any of the authors.

Informed Consent Statement does not apply.

\section{References}

1. Guan WJ, Ni ZY, Hu Y, et al. Clinical characteristics of coronavirus disease 2019 in China. N Engl J Med. 2020;382:1708-20.

2. Guo T, Fan Y, Chen M, et al. Cardiovascular implications of fatal outcomes of patients with coronavirus disease 2019 (COVID-19). JAMA Cardiol. 2020;

3. Huang $\mathrm{C}$, Wang Y, Li X, et al. Clinical features of patients infected with 2019 novel coronavirus in Wuhan, China. Lancet. 2020;395(10223):497-506.

4. Xiao F, Tang M, Zheng X, et al. Evidence for gastrointestinal infection of SARSCoV-2. Gastroenterology. 2020;158:18311833.e3.
5. Gu J, Han B, Wang J. COVID-19: gastrointestinal manifestations and potential fecal-oral transmission. Gastroenterology. 2020;158: 1518-9.

6. Zhang C, Shi L, Wang FS. Liver injury in COVID-19: management and challenges. Lancet Gastroenterol Hepatol. 2020;5:428-30.

7. Bourouiba L. Turbulent gas clouds and respiratory pathogen emissions: potential implications for reducing transmission of COVID19. JAMA. 2020;

8. van Doremalen N, Bushmaker T, Morris DH, et al. Aerosol and surface stability of SARS-CoV-2 as compared with SARS-CoV-1. N Engl J Med. 2020;

9. Richardson S, Hirsch JS, Narasimhan M, et al. Presenting characteristics, comorbidities, and outcomes among 5700 patients hospitalized with COVID-19 in the New York City area. JAMA. 2020;323:2052.

10. Chiu PWY, $\mathrm{Ng} \mathrm{SC}$, Inoue $\mathrm{H}$, et al. Practice of endoscopy during COVID-19 pandemic: position statements of the Asian Pacific Society for Digestive Endoscopy (APSDE-COVID statements). Gut. 2020;69:991-6.

11. Sultan S, Lim JK, Altayar O, et al. AGA Institute rapid recommendations for gastrointestinal procedures during the COVID-19 pandemic. Gastroenterology. 2020;

12. Patel R, Babady E, Theel ES, et al. Report from the American Society for Microbiology COVID-19 International Summit, 23 March 2020: value of diagnostic testing for SARS-CoV-2/ COVID-19. mBio. 2020;11(2)

13. He X, Lau EHY, Wu P, et al. Temporal dynamics in viral shedding and transmissibility of COVID-19. Nat Med. 2020;26:672-5.

14. Wang W, Xu Y, Gao R, et al. Detection of SARS-CoV-2 in different types of clinical specimens. JAMA. 2020;

15. Genco A, Maselli R, Frangella F, et al. Intragastric balloon for obesity treatment: results of a multicentric evaluation for balloons left in place for more than 6 months. Surg Endosc. 2015;29(8): 2339-43.

16. Repici A, Maselli R, Colombo M, et al. Coronavirus (COVID-19) outbreak: what the department of endoscopy should know. Gastrointest Endosc. 2020;92:192-7.

17. Thompson CC, Shen L, Lee LS. COVID-19 in endoscopy: Time to do more? Gastrointest Endosc. 2020;

Publisher's Note Springer Nature remains neutral with regard to jurisdictional claims in published maps and institutional affiliations. 